\title{
Sparse random matrices: the eigenvalue spectrum revisited
}

\author{
Guilhem Semerjian ${ }^{1}$ and Leticia F. Cugliandolo ${ }^{1,2}$ \\ ${ }^{1}$ Laboratoire de Physique Théorique de l'École Normale Supérieure, \\ 24 rue Lhomond, 75231 Paris Cedex 05, France \\ ${ }^{2}$ Laboratoire de Physique Théorique et Hautes Énergies, Jussieu, \\ 1er étage, Tour 16, 4 Place Jussieu, 75252 Paris Cedex 05, France \\ guilhem@lpt.ens.fr, leticia@lpt.ens.fr
}

October 22, 2018

\begin{abstract}
We revisit the derivation of the density of states of sparse random matrices. We derive a recursion relation that allows one to compute the spectrum of the matrix of incidence for finite trees that determines completely the low concentration limit. Using the iterative scheme introduced by Biroli and Monasson [J. Phys. A 32, L255 (1999)] we find an approximate expression for the density of states expected to hold exactly in the opposite limit of large but finite concentration. The combination of the two methods yields a very simple geometric interpretation of the tails of the spectrum. We test the analytic results with numerical simulations and we suggest an indirect numerical method to explore the tails of the spectrum.
\end{abstract}

LPT-ENS 02/16, LPTHE 02/10

\section{Introduction}

The list of physical applications of random matrix theory is very long. In nuclear physics, the Hamiltonians of heavy nuclei are replaced by large random matrices and their eigenvalues are associated to the energy levels. In condensed matter theory, random matrices are used to mimic the Hamiltonians of dirty conductors in the study of the metal-insulator transition. Random matrices represent the interactions in disordered magnets as spin-glasses. These physical systems are intimately related to optimization problems and the theory of random matrices also plays an important role in computer science. Random matrices are the incidence matrices of random graphs and have been extensively studied in the context of graph theory. Consequently, the development of random matrix theory and, in particular, the study of the spectrum of random matrices has a long history [ [ 1 , 2]. 
The most commonly studied random matrices are symmetric with independent identically distributed real elements. If the entries are taken from a Gaussian distribution the averaged spectrum of infinite matrices obeys the Wigner semi-circle law [1, 2, 3, 4]. For such matrices studied in the thermodynamic limit, the only way to modify this result is to consider the sparse case in which the mean number of non-zero elements on each row remains finite when the size of the matrix diverges [5]. If the elements in the random matrix represent the exchanges of a model with two-body interactions, the fully connected case corresponds to a matrix with non-vanishing entries outside the diagonal [6] while the sparse limit corresponds to a model with finite connectivity [7]. The latter problem is much harder than the former. Even if replica [8, 9] and super-symmetric [5, 10] methods have been used to compute the spectrum of dilute matrices, the derivation of a full analytic expression for it seems out of reach. A major difference between the semi-circle law and the density of states of dilute matrices lies in the presence of unbounded tails in the latter case 8, 11], which are absent in the thermodynamic limit of the former [1].

In this article we study the density of states of a sparse random matrix using a variety of techniques. We take special advantage of one recently proposed by Biroli and Monasson [12]. Even if not all our results are new, we believe that it is useful to derive them with a method that emphasizes the underlying geometric origin of the tails in the spectrum, namely, the fluctuations in the site connectivities. In a first part, we present the results stemming from cluster expansions [13], recovering results mentioned by Mirlin and Fyodorov [5] and Bauer and Golinelli [11], and showing how the density of states of any finite random matrix can be obtained in an iterative way (Section 3). Second, in Section 4 we use the replica approach, following the scheme of approximation that is based on the variational method developed in [12]. By emphasizing the links between the cluster expansion and the replica functional variational method, we give a very simple explanation of the asymptotic form of the tails of the distribution derived by Rodgers and Bray [8], and we give the first steps of its more precise investigation. In Section 5 we present the spectra obtained from the numerical diagonalisation of finite matrices. In particular, we demonstrate the importance of finite size effects as far as the dependence on the ferro or anti-ferromagnetic character of the interactions is concerned. In the conclusions we summarize our results and we show how the study of the dynamics of dilute disordered spin models are related to them [14].

\section{Definitions}

Let us consider an $N \times N$ symmetric matrix with real elements $J_{i j}$. We constrain the diagonal entries to vanish $J_{i i}=0$ and we denote $\lambda_{k}$ its $N$ eigenvalues. The elements $J_{i j}(i<j)$ are independent, identically distributed random variables with a distribution law $P\left(J_{i j}\right)$. We adopt square brackets to indicate an average over this distribution, [ $\bullet$. The central quantity under study is the average density of states

$$
\rho(\mu) \equiv\left[\frac{1}{N} \sum_{k=1}^{N} \delta\left(\mu-\mu_{k}\right)\right]
$$


in the thermodynamic limit, $N \rightarrow \infty$.

For most possible choices of $P\left(J_{i j}\right) \rho(\mu)$ is given by Wigner's semi-circle law except when one considers very sparse random matrices with a finite number of non zero elements per row in the thermodynamic limit [5]. In this case,

$$
P\left(J_{i j}\right)=\left(1-\frac{p}{N}\right) \delta\left(J_{i j}\right)+\frac{p}{N} \pi\left(J_{i j}\right)
$$

with $p$ finite. $\pi\left(J_{i j}\right)$ is a normalized distribution with average and mean square deviation of order one (this ensures a sensible thermodynamic limit) which does not contain a delta peak around zero. $p$ is the mean number of non zero elements per row, i.e. the mean connectivity of a given site.

In the following, we concentrate on a bimodal distribution

$$
\pi\left(J_{i j}\right)=a \delta\left(J_{i j}-J_{0}\right)+(1-a) \delta\left(J_{i j}+J_{0}\right)
$$

for the non-vanishing entries in the random matrix. The parameter $a$ controls the asymmetry between the "ferromagnetic" or "anti-ferromagnetic" tendency in the model. Since we can absorb the dependence on $J_{0}$ with a global rescaling of the density of states, $\rho(\mu)=\left(1 / J_{0}\right) \tilde{\rho}\left(\mu / J_{0}\right)$, we set $J_{0}=1$ henceforth.

\section{Cluster expansion}

Given a particular realization of the matrix $J$ among the random ensemble, one can associate it to a graph in a very natural way. Consider $N$ sites labeled from 1 to $N$ and draw a link between the sites $i$ and $j$ if the entry $J_{i j}$ is non zero. The value $J_{i j}$ of the interaction can be written next to the link. Two sites are called adjacent if there is a link between them, and connected if there is a path of adjacent sites from one to the other. A cluster is a set of connected sites, disconnected from all others.

These definitions make the relation between random matrices and random graphs explicit. As a matter of fact, the study of the ensemble of such graphs (forgetting the values of the interactions) is nothing but the well known random graph problem. When $p<1$ the random matrix $J$ is so sparse that all sites belong to finite clusters. At $p=1$ a percolation transition occurs and a giant cluster containing a finite fraction of the sites appears. The rest of the sites belong to finite clusters [15].

In this Section we derive a cluster expansion of the density of states, i.e. a development in powers of the mean connectivity $p$ of $\rho(\mu)$. This expansion is not valid beyond the percolation threshold; still, some results derived here give a geometrical insight into the behavior of $\rho$ for all $p$, at least in the limit $|\mu| \rightarrow \infty$.

If one orders the sites by grouping those belonging to the same cluster, the matrix $J$ acquires a block-diagonal form, each cluster being associated to one block. The density of eigenvalues for a particular realization of the ensemble, $\rho_{J}(\mu)$, can then be written as a double sum,

$$
N \rho_{J}(\mu)=\sum_{\mathcal{C}} \sum_{k_{\mathcal{C}}} \delta\left(\mu-\mu_{k_{\mathcal{C}}}\right)
$$


where the index $\mathcal{C}$ labels the clusters and $k_{\mathcal{C}}$ labels the eigenvalues of each cluster. Under this form, $\rho_{J}(\mu)$ is an additive quantity over clusters and we can apply the technique described in [13] to transform this expression into a simpler one. Indeed, clusters can be separated into ensembles with the same topology, ignoring for the moment the assignment of the values of the interactions. The averaging over different realizations of the random matrix proceeds in two steps; one first chooses the topology of the cluster, with its associated probability, and, subsequently, one averages over the values of the interactions with the distribution $\pi\left(J_{i j}\right)$. For a given cluster, once the latter average is performed, the density of states depends only on its topology. (Note that one could do so for any distribution $\pi$, even if not bimodal.) This remark allows us to rewrite the average of the sum in Eq. (4) in a more convenient manner. If we introduce an index $t$ that labels all possible topologies, $n_{t}(J)$ the number of $t$-like clusters present in a given realization of the ensemble, and $\bar{\rho}_{t}$ the average over the distribution $\pi$ of the density of states of the $t$-like cluster $\rho_{t}$, we obtain the following expression for the averaged density of eigenvalues:

$$
\rho(\mu)=\left[\rho_{J}(\mu)\right]=\sum_{t} \frac{\left[n_{t}\right]}{N} L_{t} \bar{\rho}_{t}(\mu) .
$$

We included the isolated sites associating them to the index $t=0$ and we denoted $\left[n_{t}\right]$ the average number of $t$-like clusters. As in [13] we introduce a function $X_{t}^{i}$ that takes the value 1 if the site $i$ belongs to a $t$-like cluster and 0 otherwise and we call $L_{t}$ the number of sites in a $t$-like cluster. Then

$$
n_{t}(J)=\frac{1}{L_{t}} \sum_{i} X_{t}^{i}, \quad \Rightarrow \quad \frac{1}{N}\left[n_{t}\right]=\frac{1}{L_{t}}\left[X_{t}^{1}\right]=\frac{P_{t}}{L_{t}}
$$

where $P_{t} \equiv\left[X_{t}^{1}\right]$ is the probability that a given variable belong to a $t$-like cluster. Finally,

$$
\rho(\mu)=\sum_{t} P_{t} \bar{\rho}_{t}(\mu)=\sum_{t} \frac{P_{t}}{L_{t}} \overline{\sum_{l=1}^{L_{t}} \delta\left(\mu-\mu_{l}\right)},
$$

where $\mu_{l}$ are the eigenvalues of the $t$-like cluster, $P_{t}$ is given by $P_{t}=p^{L_{t}-1} e^{-p L_{t}} K_{t}$, and $K_{t}$ is a symmetry factor [13]. The overline denotes an average over the distribution $\pi$, for fixed topology. Note that only tree-like clusters contribute to the thermodynamic limit: the probability that a site belong to a cluster containing a loop of finite length is of order $1 / N$. Reordering this series in powers of $p$, it is clear that all clusters of size $L_{t} \leq m+1$ have to be considered to obtain the expansion at order $p^{m}$.

For the bimodal $\pi\left(J_{i j}\right)$ each type of cluster contributes a sum of a finite number of delta functions to $\rho(\mu)$. The location of the delta functions are symmetrically distributed around $\mu=0$. The only task remaining in order to obtain the series expansion is to diagonalize the finite size matrices corresponding to each cluster. For instance, up to first order in $p$, one finds (for any value of $a$ ):

$$
\rho(\mu)=\delta(\mu)+\frac{p}{2}[\delta(\mu-1)-2 \delta(\mu)+\delta(\mu+1)] .
$$



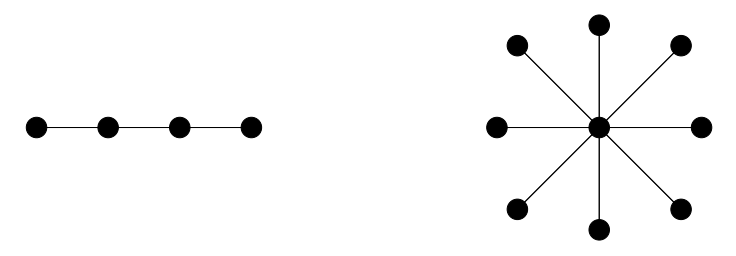

Figure 1: Two examples of finite clusters: a linear $(n=4)$ and a star-like one $(k=8)$.

There does not seem to be a straightforward way to compute the eigenvalues of an arbitrarily shaped tree-like cluster. A possible method would be to solve the integral equation arising from the replica or the super-symmetric approach perturbatively and to expand the order parameter in powers of the concentration, as explained in [5]. Another method, exposed below, is to establish recursion relations on the characteristic polynomial of finite size matrices [16].

Before getting into the general derivation, let us study two examples of simple clusters for which one can compute the eigenvalues explicitly. Consider first a linear cluster with $n$ sites (see the left panel of Fig. 1). For this structure the corresponding block in the matrix $J$ has elements \pm 1 on the two diagonals next to the main one, and 0 everywhere else. It is then a simple exercise of linear algebra to show that the $n$ eigenvalues are

$$
\mu_{l}=2 \cos \left(\frac{l \pi}{n+1}\right), \quad l=1, \ldots, n .
$$

Note that for any value of $n$, the eigenvalues belong to $[-2,2]$. The "opposite" kind of geometry is given by clusters with one site connected to $k$ neighbours (see the right panel in Fig (1). Again the matrix $J$ is readily written. One easily shows that the spectrum of such a cluster has $k-1$ vanishing eigenvalues, one eigenvalue given by $\sqrt{k}$ and another one given by $-\sqrt{k}$. This behavior is very different from the one of the linear clusters: more and more branched clusters yield larger and larger eigenvalues. Very important for the arguments developed in Section 4 is the fact that the eigenvectors associated with the eigenvalues $\pm \sqrt{k}$ have a larger magnitude on the central site than on its neighbours, with a ratio $\sqrt{k}$. Thus, for large $k$, the eigenvectors are more and more concentrated on the central site. We shall come back to this point later, when discussing the behavior of the density of states at large eigenvalues.

To derive the general recursion relation we focus on tree-like structures since loops of finite size disappear in the thermodynamic limit. We define a rooted tree $r$ as a tree with one of its sites (the root) particularized. We represent a generic rooted tree as a bubble englobing the tree, with only the root shown (see Fig. 21). If the root of the tree $r$ is connected to, say, $k$ other sites that we call its neighbours, we denote $r_{i}, i=1, \ldots, k$, the trees whose roots are the neighbours of the original root and $\epsilon_{i}$ the values of the edges that link the original root to the $k$ secondary ones (see Fig. 3).

We call $P_{r}(\lambda)$ the characteristic polynomial of the matrix associated with the rooted tree $r$, and $\tilde{P}_{r}(\lambda)$ the one of the matrix in blocks associated to the discon- 


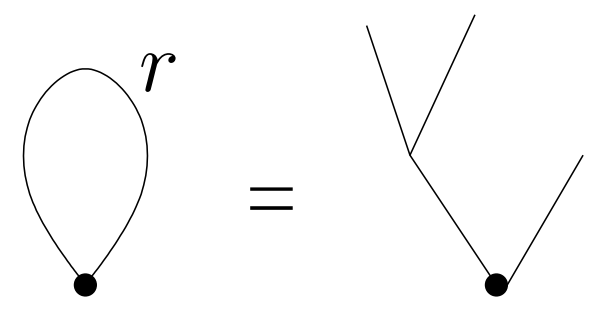

Figure 2: An example of rooted tree

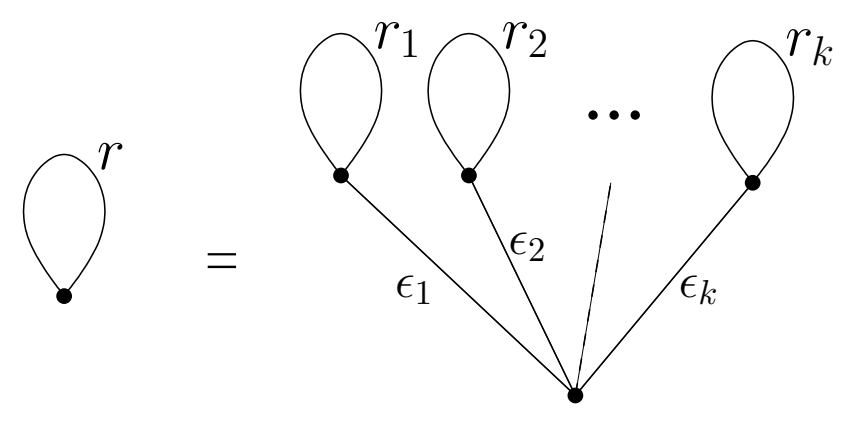

Figure 3: Decomposition of a rooted tree

nected trees obtained after deleting the root of $r$ and the edges emerging from it. We thus have

$$
\tilde{P}_{r}(\lambda)=\prod_{i=1}^{k} P_{r_{i}}(\lambda)
$$

With these definitions the recursion relation is obtained by expanding the determinant along the rows and the columns of the roots, and reads

$$
P_{r}(\lambda)=-\lambda \prod_{i=1}^{k} P_{r_{i}}(\lambda)-\sum_{i=1}^{k} \epsilon_{i}^{2} \tilde{P}_{r_{i}}(\lambda) \prod_{j \neq i} P_{r_{j}}(\lambda) .
$$

Our first remark is that the parameters $\epsilon_{i}$ appear only through their squares, so we can prove by recurrence that for any tree, the average spectrum for the bimodal distribution (3) does not depend on the value of $a$. Thus, when $p<1$ and the thermodynamic limit is taken $\rho(\mu)$ is totally independent of the ferromagnetic or anti-ferromagnetic character of the distribution. When $p>1$, loops of length of order $\ln N$ appear in the giant cluster and the argument above, relying on the tree structure of the interactions, is not valid anymore. We shall develop this point in Section 4.6.

As a simple example, let us now write down the characteristic polynomial for the two level tree sketched in Fig. 1 . The root has $k$ neighbours and each of them has $l_{i}+1$ neighbours, $i=1, \ldots, k$. If we define $L \equiv \sum_{i} l_{i}$, the characteristic polynomial is given by

$$
P(\lambda)=(-\lambda)^{L-k+1}\left[\prod_{i}\left(\lambda^{2}-l_{i}\right)-\sum_{i} \prod_{j \neq i}\left(\lambda^{2}-l_{j}\right)\right]
$$


whose roots give the $L+k+1$ eigenvalues of the corresponding matrix. For instance in the symmetric case $\left(l_{i}=l \forall i\right)$, the eigenvalues (and their degeneracies) are: 0 $(k(l-1)+1), \pm \sqrt{l}(k-1$ for each sign $)$ and $\pm \sqrt{k+l}(1$ for each sign $)$.

In principle, the relation (11) can be iterated to obtain the characteristic polynomial of any tree, and from it its eigenvalues.

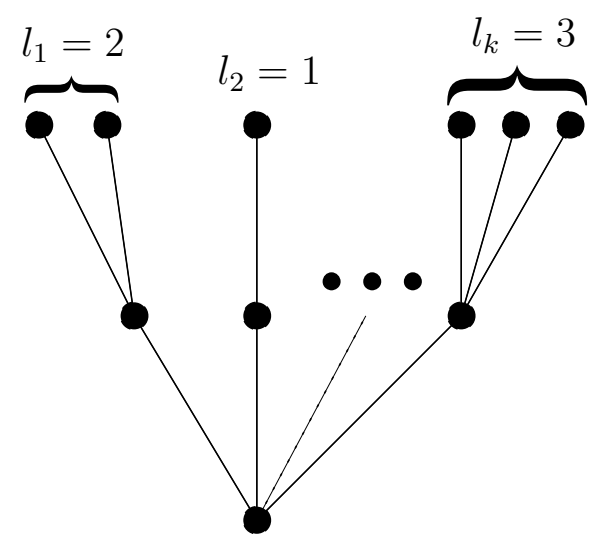

Figure 4: Two level tree

To conclude this Section, let us note than even for $p>1$, a finite fraction of sites belong to finite-size clusters. Thus, the spectra contains delta peaks located at eigenvalues of finite tree clusters for all $p$, as established in [11, 17] (it is also shown in [11] that parts of the giant cluster contribute to these delta peaks).

In the context of the gelation transition, a detailed study of the low concentration phase for related matrices was performed in [18.

\section{Replica approach}

In this Section we compute the spectrum using the replica method. We first give a qualitative argument to suggest where one can expect to find localized states. Then we describe the method and find a series of approximate expressions for $\rho(\mu)$.

\subsection{Localized eigenvectors on the Bethe lattice}

One cannot use the above cluster expansion to derive the density of states above the percolation transition $p=1$ where a giant component with a finite fraction of the sites appears in the thermodynamic limit. The giant cluster contains loops with length $O(\ln N)$ so, locally, it has a tree-like structure [15].

It is interesting to know if some eigenvectors of the sparse matrix are localized, i.e. if they have a finite fraction of their weight on a finite number of sites. A qualitative answer to this question can be given by assuming that one site has $k$ neighbours, and that all the other sites have the same connectivity $p+1$ (the average connectivity of a site having already one neighboor is $p+1$ ), with no loops. That is to say, we approximate the giant component with a Bethe lattice with a 
single "defect", the site with a connectivity that is different from the mean. It is then easy to derive a condition for the existence of an eigenvector localized on this defect and with a spherical symmetry i.e. such that its magnitude on each site only depends on the distance to the central site. For the ensemble of matrices under consideration, one finds the condition: $k \geq 2 p$. Thus, we expect localized eigenstates to appear around sites with connectivities much larger than the mean. Note that for the Laplacian of a random graph (instead of the incidence matrix considered here) the same argument leads to $|k-p| \geq \sqrt{p}$ [12] for the localization condition of eigenstates; the required deviation of the local connectivity from the mean is less stringent in this case.

This argument can be refined by considering a site with $k$ neighbours, each having $l+1$ neighbours, and all other sites having the mean connectivity $p+1$. Then the condition for the existence of a localized eigenstate is : $k+2 l \geq 4 p$. Of course if one takes $l=p$, the above condition is recovered. If $l$ is slightly lower than the mean, the condition on $k$ becomes more and more demanding. Conversely, if $l$ is slightly higher than the mean, the condition on $k$ is relaxed. One can expect the latter case to be privileged in random graphs for which the distribution of connectivities is Poissonian: sites with connectivity of order twice the mean are rare, but their neighbours are likely to have a connectivity slightly above the mean.

\subsection{Method and definitions}

Field theories, either replicated [3] or super-symmetric [2, 4], have been used to determine the average spectrum of random matrices. For sparse matrices, the result is expressed in terms of the solution to an integral equation, which has proven too difficult to be solved exactly. Approximate results, either perturbative or non perturbative [8] with respect to the inverse concentration $1 / p$ have been obtained. We shall revisit this problem using the replica approach and the scheme of approximation developed by Biroli and Monasson [12] (see also [19] for a similar scheme). This method will allow us to unveil the geometric origin of the tails in the distribution and to compare with results established above for finite size clusters. For simplicity, we focus on the symmetric bimodal distribution introduced in (3) with $a=1 / 2$. We come back to the dependence on $a$ in Section 4.6.

The normalized density of states for a particular realization of disorder can be computed in terms of the resolvent [1],

$$
\rho_{J}(\mu)=\frac{1}{N \pi} \operatorname{Im} \operatorname{Tr}\left[J_{i j}-(\mu+i \epsilon) \delta_{i j}\right]^{-1}=\left.\frac{2}{N \pi} \operatorname{Im} \frac{\partial}{\partial \mu} \ln Z(\mu)\right|_{\mu+i \epsilon},
$$

with $\epsilon>0$ and infinitesimal, and

$$
Z(\mu)=\int \prod_{i} d \phi_{i} \exp \left(\frac{i \mu}{2} \sum_{i} \phi_{i}^{2}-\frac{i}{2} \sum_{i j} J_{i j} \phi_{i} \phi_{j}\right) .
$$

The average over disorder, $\left[\rho_{J}(\mu)\right]$, is evaluated by introducing replicas to average the $\operatorname{logarithm}, \lim _{n \rightarrow 0} \ln \left[Z^{n}\right]=n[\ln Z]$. A very useful way to study the mean field 
theory of dilute systems [20] relies on introducing a function $c(\vec{\phi})$ that measures the fraction of sites with a field $\vec{\phi}_{i}$ equal to a chosen value $\vec{\phi}$, the vector denoting the $n$-dimensional replica space,

$$
c(\vec{\phi}) \equiv \frac{1}{N} \sum_{i} \delta\left(\vec{\phi}-\vec{\phi}_{i}\right) .
$$

Thus,

$$
\rho(\mu)=\left.\lim _{n \rightarrow 0} \frac{2}{n N \pi} \operatorname{Im} \frac{\partial}{\partial \mu} \ln \left[Z^{n}\right]\right|_{\mu+i \epsilon}
$$

with

$$
\begin{aligned}
{\left[Z^{n}\right] } & =\int \mathcal{D} c(\vec{\phi}) \exp \left(-N S_{\mathrm{EFF}}(c(\vec{\phi}))\right) \\
S_{\mathrm{EFF}}(c(\vec{\phi})) & =\int d \vec{\phi} c(\vec{\phi}) \ln c(\vec{\phi})-\frac{i \mu}{2} \int d \vec{\phi} c(\vec{\phi}) \vec{\phi}^{2}+H_{\mathrm{EFF}}(c(\vec{\phi}))
\end{aligned}
$$

where the integration is taken only over normalized $c(\vec{\phi})$. The effective Hamiltonian is defined as

$$
\exp \left(-N H_{\mathrm{EFF}}\right)=\left[\exp \left(-\frac{i}{2} \sum_{i j} J_{i j} \phi_{i} \phi_{j}\right)\right]_{J}
$$

and for the ensemble of symmetric sparse random matrices with a bimodal symmetric distribution it reads

$$
-H_{\mathrm{EFF}}(c(\vec{\phi}))=-\frac{p}{2}+\frac{p}{2} \int d \vec{\phi} d \vec{\psi} c(\vec{\phi}) c(\vec{\psi}) \cos (\vec{\phi} \cdot \vec{\psi})
$$

In the limit of large sizes, $N \rightarrow \infty$, the path integral in Eq. (17) can be computed with the saddle-point method and it is dominated by the neighbourhood of the extreme, $c^{\mathrm{SP}}(\vec{\phi})$, of the effective action $S_{\mathrm{EFF}}$, which satisfies:

$$
c(\vec{\phi})=\mathcal{N} \exp \left[\frac{i}{2} \mu \vec{\phi}^{2}-\frac{\delta H_{\mathrm{EFF}}}{\delta c(\vec{\phi})}\right]
$$

with $\mathcal{N}$ a normalization constant.

Then, the averaged density of states reads

$$
\rho(\mu)=\lim _{n \rightarrow 0} \frac{1}{\pi n} \operatorname{Re} \int d \vec{\phi} c^{\mathrm{sP}}(\vec{\phi}) \vec{\phi}^{2} .
$$

Even with the assumption of invariance of $c(\vec{\phi})$ with respect to rotations in the $n$-dimensional replica space, it has proven too difficult to solve the saddle point equation explicitly. A numerical solution of a similar equation was given [21] in the context of the study of the instantaneous normal modes in a liquid. 


\subsection{Effective medium approximation}

The first step of the approximation scheme [12 consists in solving the problem variationally, i.e. by restricting the original problem of extremization to the particular Gaussian subspace of the functions $c(\vec{\phi})$. This first step is called the "effective medium approximation" (EMA) since it amounts to assuming that all the sites are equivalent and play the same role. Thus one replaces $c(\vec{\phi})$ with a Gaussian Ansatz

$$
c^{\mathrm{EMA}}(\vec{\phi})=(2 \pi i \sigma(\mu))^{-n / 2} \exp \left(-\frac{\vec{\phi}^{2}}{2 i \sigma(\mu)}\right),
$$

with $\sigma(\mu)$ the variational function determined by the stationarity condition $\delta S_{\mathrm{EFF}} / \delta \sigma(\mu)=0$. After some algebra, we find that $\sigma=\sigma(\mu)$ is given by the cubic equation

$$
\sigma^{3}+\frac{p-1}{\mu} \sigma^{2}-\sigma+\frac{1}{\mu}=0 .
$$

Among the three roots of the equation, one has to choose the one with $\operatorname{Im} \sigma(\mu+i \epsilon)<0$ for the integrals to converge. The average density of states reads $\rho^{\mathrm{EMA}}(\mu)=-1 / \pi \operatorname{Im} \sigma(\mu+i \epsilon)$. It is easy to check that these equations yield the correct Wigner semi-circle law in the fully connected limit, when $J_{0} \rightarrow J_{0} / \sqrt{N}$ and $p \rightarrow N$. The EMA is exact in this case.

In the following we focus on the case $p>1$. For $p<1$, the sites belong to finite size clusters and they are very heterogeneous in nature so we do not expect the EMA to yield accurate results. Moreover the cluster expansion is exact in this regime and it is enough to solve the problem completely. The approach used here should be valid for $p$ large but finite, as the larger the value of $p$, the smaller the fraction of sites in finite size clusters.

Solving the cubic equation, one finds that for $|\mu| \leq \lambda_{c}$ the density of states is given by the continuous function

$$
\begin{aligned}
\rho^{\mathrm{EMA}}(\mu)= & \frac{\sqrt{3}}{2 \pi} \sqrt[3]{-\left(\frac{p-1}{3 \mu}\right)^{3}-\frac{p+2}{6 \mu}+\sqrt{\frac{\left(\lambda_{c}^{2}-\mu^{2}\right)\left(\mu^{2}+\alpha^{2}\right)}{27 \mu^{4}}}} \\
& -\frac{\sqrt{3}}{2 \pi} \sqrt[3]{-\left(\frac{p-1}{3 \mu}\right)^{3}-\frac{p+2}{6 \mu}-\sqrt{\frac{\left(\lambda_{c}^{2}-\mu^{2}\right)\left(\mu^{2}+\alpha^{2}\right)}{27 \mu^{4}}}}
\end{aligned}
$$

and vanishes outside the band $\left[-\lambda_{c}, \lambda_{c}\right]$, with

$$
\lambda_{c}=\sqrt{\frac{-p^{2}+20 p+8+\sqrt{p(p+8)^{3}}}{8}}, \quad \alpha^{2}=\frac{p^{2}-20 p-8+\sqrt{p(p+8)^{3}}}{8},
$$

see the solid curve in Fig. 5. The density of states in the EMA vanishes at $\pm \lambda_{c}$ as a square root, $\rho^{\mathrm{EMA}}(\mu) \sim\left(\lambda_{c}-|\mu|\right)^{1 / 2}$, just as for a semi-circle law. Expanding Eq. (24) 
in powers of $1 / p$ one recovers the first orders of the $1 / p$ expansion of Rodgers and Bray [8]:

$$
\begin{aligned}
\lambda_{c} & \sim 2 \sqrt{p}\left(1+\frac{1}{2 p}\right), \\
\rho(\mu) & \sim \frac{2}{\pi \lambda_{c}^{2}} \sqrt{\lambda_{c}^{2}-\mu^{2}}\left[1+\frac{1}{p}\left(1-\frac{4 \mu^{2}}{\lambda_{c}^{2}}\right)\right] .
\end{aligned}
$$

It would be interesting to check if the EMA and the perturbative solution of these authors lead to the same density of states at all orders in the expansion in powers of $1 / p$. If this were true, the EMA would be the exact resummation of the perturbative solution in 8 .

Note that at this level of the approximation scheme there are no states outside the band $\left[\lambda_{c}, \lambda_{c}\right]$, which is consistent with the qualitative argument given in Section 4.1: localized states with high eigenvalues are due to fluctuations of the local connectivity, whereas, by definition, the EMA assumes a uniform local connectivity.

\subsection{Single defect approximation}

To go beyond the EMA, it is useful to write the saddle point equation $(20)$ on $c(\vec{\phi})$ in the following form

$$
c(\vec{\phi})=\eta e^{\frac{i}{2} \mu \vec{\phi}^{2}} \sum_{k=0}^{\infty} \frac{e^{-p} p^{k}}{k !}\left[\int d \vec{\psi} c(\vec{\psi}) \cos (\vec{\phi} \cdot \vec{\psi})\right]^{k},
$$

with $\eta$ a normalization constant tending to 1 as $n \rightarrow 0$. This equation has the following geometric interpretation: any chosen site has $k$ neighbours with probability $\exp (-p) p^{k} / k$ ! and each of them interact with the central site through the effective Hamiltonian. The self consistent equation is obtained by imposing the equality of the distribution of effective fields $c(\vec{\phi})$ for all sites.

In the single defect approximation, one uses the above saddle-point equation in an iterative form, i.e one inserts the result of the Gaussian approximation in the right-hand side of Eq. (28). This means that one allows the connectivity of a given site to fluctuate but its neighbours are treated as part of the effective medium. The procedure yields

$$
\rho^{\mathrm{SDA}}(\mu)=\sum_{k=0}^{\infty} \frac{e^{-p} p^{k}}{k !}\left(-\frac{1}{\pi}\right) \operatorname{Im} \frac{1}{\mu+i \epsilon-k \sigma(\mu+i \epsilon)} .
$$

For $\mu \in\left[-\lambda_{c}, \lambda_{c}\right]$, the SDA density of states is different from the EMA one; yet it still vanishes at $\pm \lambda_{c}$ as a square root. In Fig. 5 we show the central band of the density of states in the EMA and the SDA for $p=10$; for comparison, we include a semi-circle with the same support. It is clear from the figure that the main modification introduced by the SDA is concentrated around $\mu \sim 0$.

Outside the band, delta peaks appear when the denominator of the above expression vanishes. They are located at $\pm \mu_{k}$, with

$$
\sigma\left(\mu_{k}\right)=\frac{\mu_{k}}{k} \quad, \quad\left|\mu_{k}\right| \geq \lambda_{c}
$$




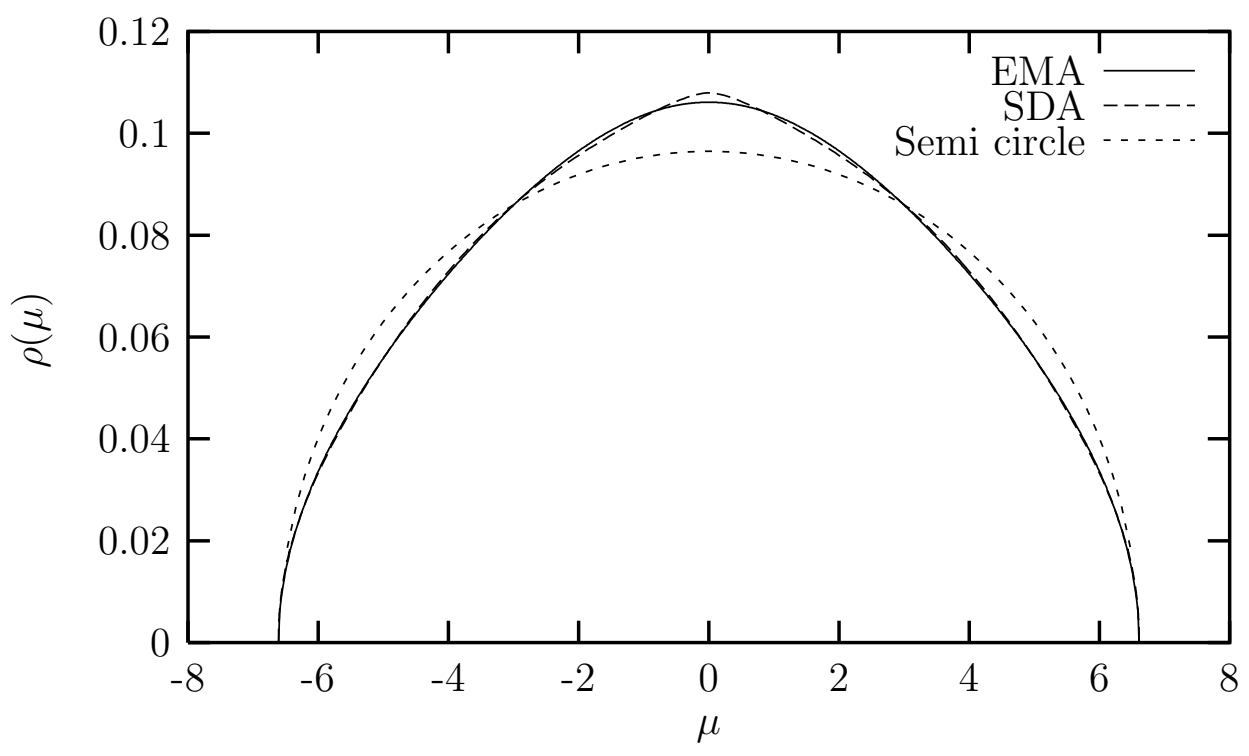

Figure 5: Extended part of the density of states for $p=10$, in the EMA and SDA $\left(\lambda_{c} \approx 6.60\right)$. A semi-circle of same width is shown for comparison.

and the weight of the peak at $\mu= \pm \mu_{k}$ is

$$
\frac{e^{-p} p^{k}}{k !} \frac{1}{1-k \sigma^{\prime}\left(\mu_{k}\right)} \text {. }
$$

As $\sigma$ is a real uneven decreasing function of $\mu$ for $|\mu| \geq \lambda_{c}$, there is a minimum value of $k$ for which Eq. (30) has a solution. A sufficiently large fluctuation of the local connectivity is necessary for localization, as predicted by the qualitative argument given in Section 4.1. For instance, for $p=10$, the first peak is found for $k_{\text {min }}=24$. One can check that $k_{\text {min }} \sim 2 p$ when $p \rightarrow \infty$ and the condition derived by the qualitative argument of Section 4.1 is recovered in this limit.

Since the peaks extend all the way up to $|\mu| \rightarrow \infty$ (as $k$ grows, the peaks move away from $\lambda_{c}$ ) we can now work out the asymptotic behaviour of the density of states. Far from the continuous band, i.e. when $k \rightarrow \infty$ and consequently $|\mu| \rightarrow \infty$, we have $\sigma(\mu) \sim 1 / \mu$. The peaks are thus located at $\mu_{k} \sim \pm \sqrt{k}$ and their weight is $\exp (-p) p^{k} /(2 k !)$ for both signs. In this limit, the distance between the peaks decreases, since $(\sqrt{k}-\sqrt{k-1}) \sim 1 /(2 \sqrt{k}) \rightarrow 0$ and one can propose a continuous approximation to describe the tails of the distribution:

$$
\rho^{\mathrm{SDA}}(\sqrt{k})(\sqrt{k}-\sqrt{k-1}) \sim \frac{1}{2} \frac{e^{-p} p^{k}}{k !} .
$$

After expanding for large $k$ and changing variables $k \rightarrow \mu^{2}$, one obtains the asymptotic form

$$
\rho^{\mathrm{SDA}}(\mu) \sim \frac{1}{\sqrt{2 \pi}} e^{-p} \exp \left[-\mu^{2} \ln \left(\frac{\mu^{2}}{p e}\right)\right] \quad \mu \gg \lambda_{c} .
$$

This result was obtained by Rodgers and Bray in [8], after an involved non perturbative treatment of an integral equation following a method proposed in [22]. 
Let us emphasize the very simple geometric interpretation of this asymptotic form unveiled by the present derivation. A given site in a random graph has $k$ neighbours with probability $\exp (-p) p^{k} / k$ !. For large $k$, there are two eigenvectors localized on it, with eigenvalues $\pm \sqrt{k}$. It is then not surprising that in the limit of large $k$, one finds the same eigenvalues as for a star-like finite cluster. When $k$ grows, the eigenvector is more and more concentrated on the central site and the eigenvalue is less and less sensitive to the environment of the first neighbours; if they are disconnected from the rest of the sites, as for finite clusters, or inside the giant cluster is not important in this limit [23.

We would like to stress a similarity with a result in [11]. These authors express the moments of $\rho(\mu)$ as polynomials in $p$ and they provide a bound for the coefficients of these polynomials. Their lower bound is expressed in terms of Stirling numbers and polynomials that are the moments of the distribution $f_{p}(x)=\frac{e^{-p}}{2} \sum_{k=0}^{\infty} \frac{p^{k}}{k !}(\delta(x-\sqrt{k})+\delta(x+\sqrt{k}))$ that we argued to be the asymptotic form of the eigenvalue spectrum. Moreover, Stirling numbers also arise when considering star-like geometries via the enumeration of particular walks on these trees. There should be a deep connection between these two approaches beyond this formal similarity.

\subsection{Two level approximation}

Let us now briefly explain how to improve on the above results. The next step in the approximation scheme consists in iterating the saddle point equation once more, by inserting $c^{\mathrm{SDA}}(\vec{\phi})$ in the right hand side of Eq. (28). The spectrum in the interval $\left[-\lambda_{c}, \lambda_{c}\right]$ is again modified with respect to the ones found in the EMA and SDA. However, it still vanishes at $\pm \lambda_{c}$ and keeps a more or less similar form. Outside this band, peaks indexed by $k, l_{1}, \ldots, l_{k}$, with $k$ and $l_{i}$ integers, appear for values of $\mu$ that are solutions to the equation:

$$
1=\sum_{i=1}^{k} \frac{1}{\mu^{2}-l_{i} \mu \sigma(\mu)} \quad \text { with }|\mu| \geq \lambda_{c} .
$$

This corresponds to considering a central site having $k$ neighbours, each having $l_{i}+1$ neighbours, the latter being treated as part of the effective medium. One has thus a more precise description of the environment of the given site since its first neighbours are treated exactly. Again, if one considers the limit $|\mu| \rightarrow \infty$, for which $\sigma(\mu) \sim 1 / \mu$, Eq. (34) yields the roots of the characteristic polynomial of a finite cluster with the same geometry, see Eq. (12). In this limit, the part of the graph under consideration is not sensitive to the rest of the sites.

For the same reasons explained at the SDA level, there are minimum values of $k$, $\left\{l_{i}\right\}$, for a solution to Eq. (34) to exist. As predicted by the qualitative argument, there can be localized states with $k$ less than $k_{\text {min }}$ of the SDA, provided the $l_{i} \mathrm{~S}$ are not too small. For instance, if $p=10$, there are solutions with $k=20$ and all the $l_{i}$ s equal to 13 .

Even if we have not derived an explicit asymptotic expression of the density of state at this level of approximation, we believe that the qualitative features of $\rho^{\mathrm{SDA}}(\mu)$ 
will not be modified. Indeed, large eigenvalues are due to sites having a connectivity much larger than the mean and these are rare events. Configurations in which the connectivity of the first neighbours also differs considerably from the one of the effective medium have even lower probability, they should then be negligible and yield only a small correction. Yet, it would be interesting to evaluate it and to check if it is consistent with the next terms of the expansion which, in principle, can be computed from the non perturbative method in [8].

\subsection{Asymmetric distribution}

In this section we discuss the influence of the asymmetry parameter $a$ that quantitifies the ferromagnetic or anti-ferromagnetic character of the interactions, see Eq. (3).

We have shown rigorously that as long as the underlying graph is tree-like, the parameter $a$ has strictly no influence on the spectrum. Thus, when $p<1$, in the thermodynamic limit, $\rho(\mu)$ is strictly independent on $a$.

When $p>1$, one can show that $a$ has no effect on the iterative scheme of resolution of the saddle point equation. Indeed, for $a \neq 1 / 2$, the effective Hamiltonians becomes:

$$
-H_{\mathrm{EFF}}(c(\vec{\phi}))=-\frac{p}{2}+\frac{p}{2} \int d \vec{\phi} d \vec{\psi} c(\vec{\phi}) c(\vec{\psi})\left(a e^{-i \vec{\phi} \cdot \vec{\psi}}+(1-a) e^{i \vec{\phi} \cdot \vec{\psi}}\right) .
$$

Since $c^{\mathrm{EMA}}(\vec{\phi})$ is even with respect to $\vec{\phi} \rightarrow-\vec{\phi}$, Eq. (23) on $\sigma(\mu)$ is not modified. Then $c^{\mathrm{SDA}}(\vec{\phi})$ is independent on $a$ and even so, at all orders of the iteration, the order parameter, and thus the spectrum, do not depend on $a$.

The independence of $\rho(\mu)$ on $a$ was conjectured in 10 and shown rigorously in 11]: the moments of the spectrum do not depend on $a$, and their growth is sufficiently slow to determine the density of states. However, we observed numerically a strong dependence on $a$ in finite size matrices (see Section 5). As practical applications of random matrix theory are often confined to small matrix sizes, this cannot be neglected.

\section{Numerical results}

In this Section we present the spectra obtained from the exact numerical diagonalisation of finite size random matrices.

In Fig 6] we plot the average density of states, computed on 500 samples of $2000 \times 2000$ matrices, with mean connectivity $p=10$ and a symmetric distribution of the matrix elements, $a=1 / 2$. The agreement with the SDA prediction is very good in most of the spectrum. However, there is a tail extending beyond $\lambda_{c}$, shown in the inset. We have checked that this tail was not due to finite-size effects by repeating the diagonalization for different values of $N$. We have also checked that eigenvalues in the tail are due to localized states, by computing the inverse participation ratio for these states, which does not scale as $1 / N$ in the large $N$ limit. However, the height of the peaks predicted within the SDA is much too small to explain this tail 
quantitatively. We believe that successive iterations of the approximation scheme are necessary to describe it, by taking into account the "cooperative effect" of neighbours of a site of large connectivity having connectivities slightly higher than the mean. As argued in Section 19 we expect the SDA to be correct at large eigenvalues. However, the weight in the asymptotic tail is so small that it would require very large matrix sizes and a very large number of samples to be observed. Because of the high computational cost of matrix diagonalization, it seems impossible to study this phenomenon directly. In the conclusion we propose an alternative method to analyse it.

We have also studied numerically the influence of the asymmetry parameter $a$ on the average density of states. In Fig. 0 we plot the average density of states, computed on 2000 samples of $800 \times 800$ matrices, with mean connectivity $p=10$ and a purely ferromagnetic distribution, $a=1$. We find a very similar spectrum in the region $\left[-\lambda_{c}, \lambda_{c}\right]$ with the addition of a separated bump at larger eigenvalues. The total weight in the bump is, up to numerical precision, $1 / N$ (we have checked this scaling for different values of $N$ ), suggesting that for each realization of the matrix, the largest eigenvalue is in the bump. This assumption was confirmed directly, by treating separately the largest eigenvalue of each sample.

For $N=800$ and $p=10$ we found that the location of the largest eigenvalue decreases smoothly from $a=1$ to $a \approx 0.65$, where it approximately approaches the edge of the $a=0.5$ distribution. This observation is not contradictory with the previous statements on the independence on $a$ of the density of states in the thermodynamic limit, as this effect is of order $1 / N$. A detailed study of the $N$ and $a$ dependence is delicate and it goes beyond the scope of this work. Yet, it is important to keep in mind this effect for realistic implementations where finite size effects are important.

\section{Conclusions}

The study of random matrices is a very rich field. For sparse matrices, there has been interest in different ensembles (Laplacians [9, 12, 21], incidence matrices [8], "real-world" graphs [24], banded matrices [25]), and in different properties (large eigenvalue tails, behaviour around small eigenvalues, quantum/classical percolation [26]). In this paper we focused on the specific problem of the large eigenvalue tails. We studied the properties of finite-size clusters and derived recursion relations on characteristic polynomials. This allows one to solve exactly the problem for low concentrations $p$. In addition we used a replica variational method 12 to study the large $p$ limit. The combination of these two methods gives a very simple geometric content to the asymptotic form of the eigenvalue tails previously obtained with involved calculus [ [B] . It is worth mentioning though a drawback of the method we used: it is not straightforward to estimate the range of validity of the result and to bound the corrections that successive levels of iterations generate.

As mentioned in Section 5, the direct exploration of the tails in the eigenvalue spectrum by means of numerical diagonalisation is a very difficult task, as these tails 


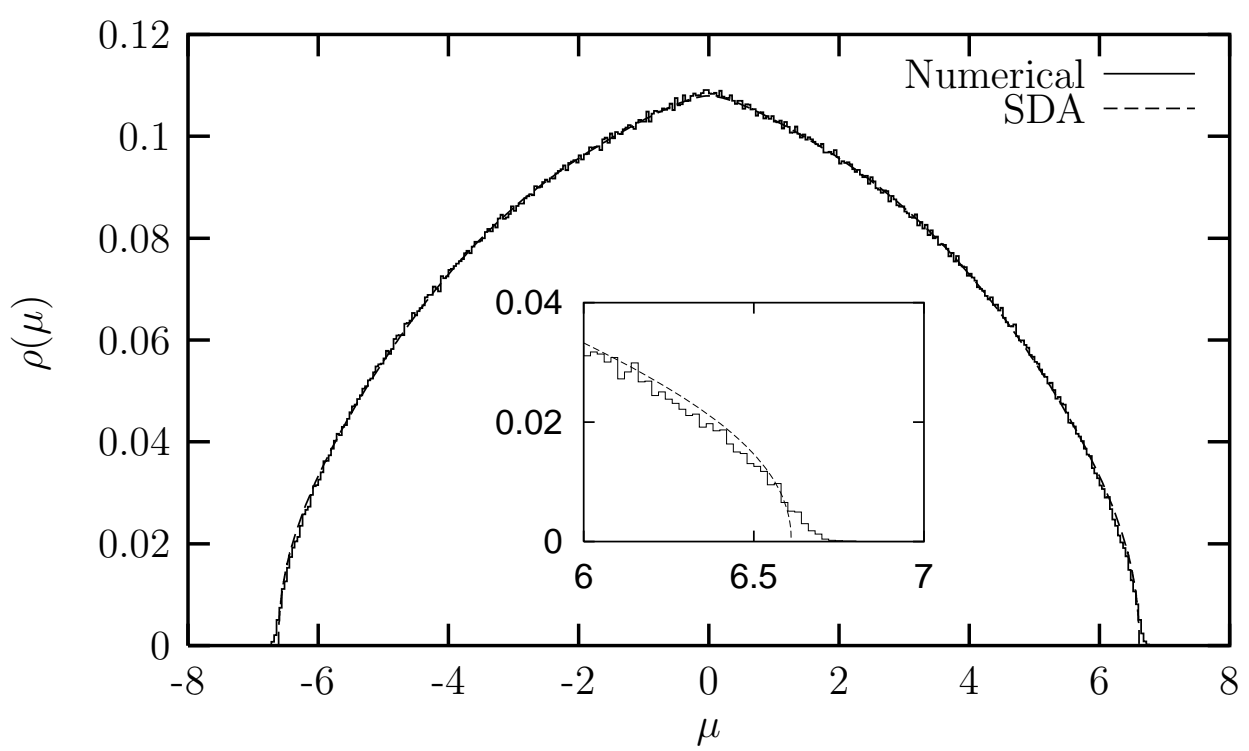

Figure 6: Density of states averaged over 500 samples for $p=10, a=1 / 2$, the matrix size is $N=2000$. The dashed curve represents the SDA result for this value of $p$ and it is almost totally superposed to the numerical data apart from a small deviation near the edges, shown in the inset.

are due to very rare events. In a forthcoming paper [14 we shall use an indirect method that might make this study easier. Namely, we shall consider the dynamics of a spherical model with finite connectivity interactions, whose dynamics is directly related to the eigenvalue spectrum considered here. The dynamics in the long time limit is controled by the large eigenvalue behaviour of the density of states and its study will hence give us information about the tails of the spectrum itself.

Acknowledgments

Very useful discussions with Giulio Biroli and Rémi Monasson are gratefully acknowledged. GS and LFC acknowledge financial support from the ACI "Algorithmes d'optimisation et systèmes désordonnés quantiques". LFC acknowledges financial support from the research contract between the CNRS (France) and CONICET (Argentina), thanks F. A. Schaposnik and the Physics Department at the Universidad Nacional de La Plata, Argentina, for hospitality during the last part of this work and the Guggenheim Foundation. LFC is associate researcher at ICTP, Trieste, Italy.

\section{References}

[1] M. L. Mehta, Random matrices (Academic Press, 1991). 


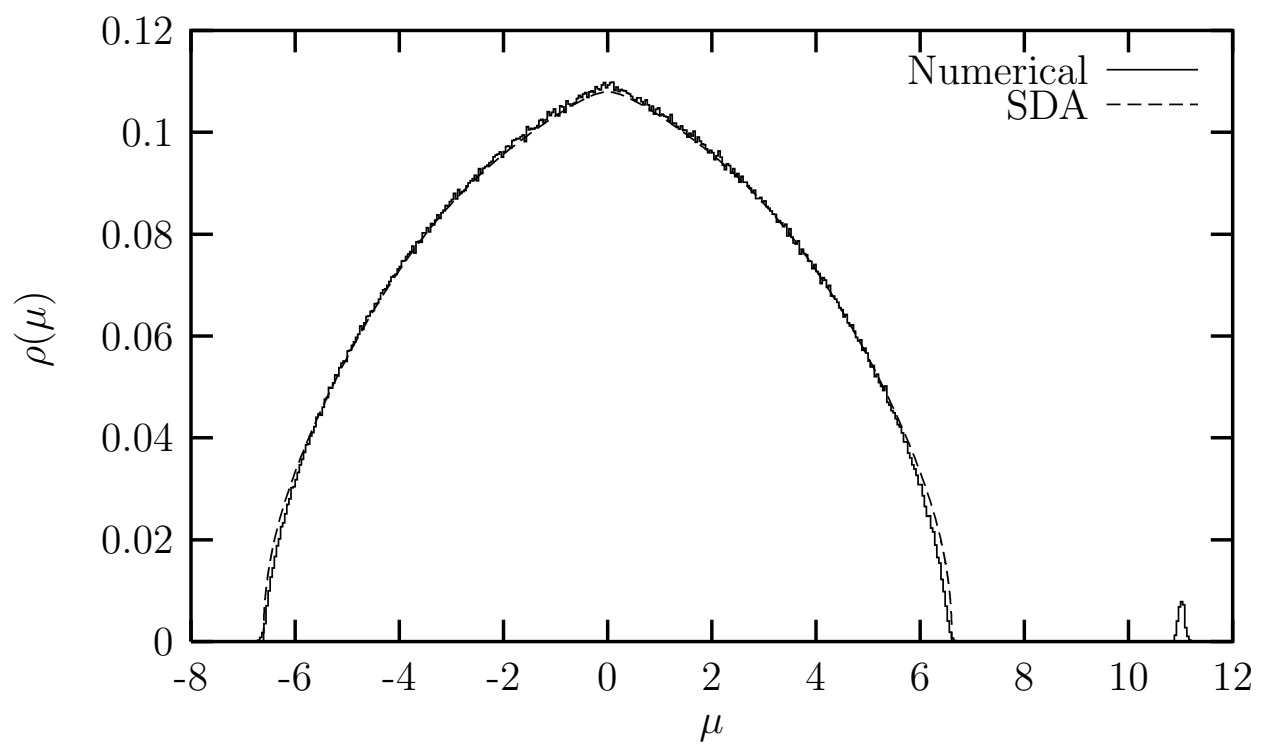

Figure 7: Density of states averaged over 2000 samples for $p=10, a=1$ and a matrix size $N=800$, with a dashed curve the SDA result for this value of $p$. Note the ferromagnetic bump at $\mu \approx 11$.

[2] J. J. M. Verbaarschot, H. A. Weindermüller and M. R. Zirnbauer, Phys. Rep. 129, 367 (1985). T. Guhr, A. Müller-Groeling and H. A. Weindermüller, Phys. Rep. 299, 189 (1998).

[3] S. F. Edwards and R. C. Jones, J. Phys. A 9, 1595 (1976).

[4] K. B. Efetov, Supersymmetry in disorder and chaos, (Cambridge Univ. Press, New York, 1997).

[5] A. D. Mirlin and Y. V. Fyodorov, J. Phys. A 24, 2273 (1991).

[6] D. S. Sherrington and S. Kirkpatrick, Phys. Rev. Lett. 35, 1792 (1975).

[7] L. Viana and A. J. Bray, J. Phys. C 18, 3037 (1985).

[8] G. J. Rodgers and A. J. Bray, Phys. Rev. B 37, 3557 (1988).

[9] A. J. Bray and G. J. Rodgers, Phys. Rev. B 38, 11461 (1988).

[10] G. J. Rodgers and C. de Dominicis, J. Phys. A 23, 1567 (1990).

[11] M. Bauer and O. Golinelli, J. Stat. Phys. 103, 301 (2001).

[12] G. Biroli and R. Monasson, J. Phys. A 32, L255 (1999).

[13] G. Semerjian and L. F. Cugliandolo, Phys. Rev. E 64, 036115 (2001).

[14] G. Semerjian and L. F. Cugliandolo, in preparation. 
[15] B. Bollobàs, Random graphs, Academic Press, London, 1985.

[16] See also B. Derrida and G. J. Rodgers, J. Phys. A 26, L457 (1993).

[17] See also S. Kirkpatrick and T. P. Eggarter, Phys. Rev. B 6, 3598 (1972).

[18] K. Broderix, T. Aspelmeier, A. K. Hartmann and A. Zippelius, Phys. Rev. E 64, 021404 (2001).

[19] A. J. Bray and D. Huifang, Phys. Rev. B 40, 6980 (1989).

[20] R. Monasson, Phil. Mag. B 77, 1515 (1998).

[21] A. Cavagna, I. Giardina and G. Parisi, Phys. Rev. Lett. 83, 108 (1999).

[22] Y. Kim and A.B. Harris, Phys. Rev. B 31, 7393 (1985).

[23] Geometric arguments were also given for a different ensemble of matrices in [9].

[24] I. J. Farkas, I. Derényi, A.-L. Barabási and T. Vicsek, Phys. Rev. E 64, 026704 (2001)

[25] Y. V. Fyodorov, O. A. Chubykalo, F. M. Izrailev and G. Casati, Phys. Rev. Lett. 76, 1603 (1996).

[26] S. N. Evangelou and E. N. Economou, Phys. Rev. Lett. 68, 361 (1992). 